\title{
Prozesskostenrisiko vs. Anspruch auf Zugang zum Recht
}

Beda Stähelin *

Die Ungewissheit über den Verfahrensausgang gehört zur Natur des Zivilprozesses. Die Beschreitung des Rechtswegs bedeutet somit unweigerlich auch die Inkaufnahme des Risikos, im Prozess zu unterliegen. Mit der Verwirklichung dieses Risikos einher geht in aller Regel die Aufbürdung der Prozesskosten. Die drohende finanzielle Last des Prozessverlusts bzw. - vereinfacht ausgedrückt - das Prozesskostenrisiko hält viele Rechtsuchende von der Anrufung des Richters ab. Es zeitigt mithin eine abschreckende Wirkung, welche die Inanspruchnahme der staatlichen Rechtspflege erschwert. Der vorliegende Beitrag befasst sich mit der Frage nach der Vereinbarkeit dieser «Kostenhürde» mit dem grundrechtlichen Anspruch auf Zugang zum Recht.

I. Einleitung. 21

II. Anspruch auf Zugang zum Recht .......................................................... 21

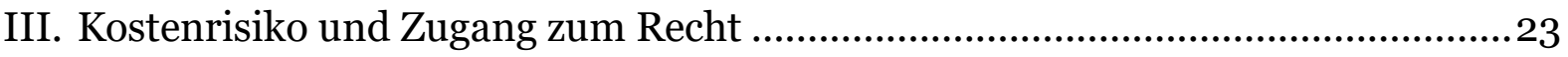

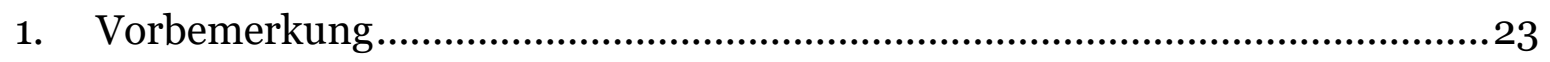

2. Zugangshemmender Effekt des Kostenrisikos ...........................................24

3. Auswirkungen in der Praxis .............................................................. 25

4. Folgerung: Kostenrisiko als faktische Rechtswegsperre im Einzelfall ............27

5. Nutzen des zugangshemmenden Effekts des Kostenrisikos .........................28

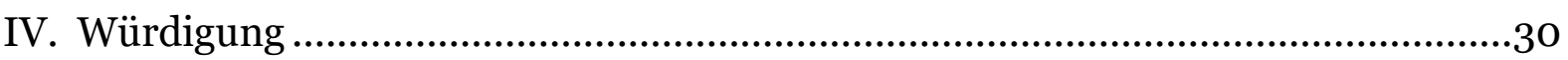

Zitiervorschlag: Beda Stähelin, Prozesskostenrisiko vs. Anspruch auf Zugang zum Recht, in: sui-generis 2018, S. 20

URL: $\quad$ sui-generis.ch/56

DOI: $\quad$ https://doi.org/10.21257/sg.56

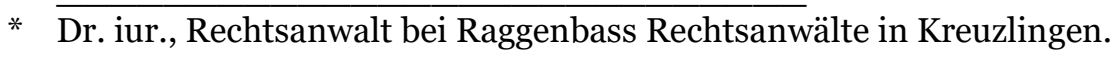




\section{Einleitung}

1 Vor Gericht und auf hoher See ist man in Gottes Hand - Selbstverständlich ist diese Redewendung überspitzt und in ihrer Absolutheit falsch. In Anbetracht der überragenden Bedeutung einer funktionierenden Justiz für den Rechtsstaat erscheint die Unterstellung, dass der Ausgang eines Zivilprozesses letztlich ein Zufallsprodukt sei, geradezu als ketzerisch. Gleichwohl lässt sich nicht in Abrede stellen, dass dem Sprichwort ein Funken oder zumindest ein Fünklein Wahrheit innewohnt. Der Verlauf eines Gerichtsverfahrens ist stets von verschiedensten mehr oder weniger bis gar nicht beeinflussbaren Faktoren abhängig, weshalb auch bei einer hervorragenden Ausgangslage eine gewisse Restunsicherheit bestehen bleibt. Das Risiko des Prozessverlusts kann somit selbst bei optimalen Voraussetzungen bestenfalls minimiert, aber nie gänzlich eliminiert werden - der «wasserdichte» Fall existiert schlicht nicht. Wer folglich den Rechtsweg beschreitet, nimmt dadurch stets auch das Risiko in Kauf, am Ende als (Prozess-)Verlierer dazustehen.

Die Verwirklichung dieses Risikos bezahlt man anders als beim Schiffbruch nicht gerade mit seinem Leben, aber immerhin mit der Last der Rechtsverfolgungskosten: Wer im Verfahren unterliegt, vermag nicht nur den geltend gemachten Anspruch nicht durchzusetzen, sondern muss darüber hinaus auch noch die Prozess- und Parteikosten berappen. Je nach Fall und Verfahrensverlauf können diese Kosten sogar den Wert des Streitgegenstands übertreffen. In Anbetracht dieses Kostenrisikos und der nie gänzlich vermeidbaren Möglichkeit, im Prozess zu unterliegen, gebietet die Vernunft nicht sel- ten, auf die Anstrengung eines Gerichtsverfahrens zu verzichten - mitunter auch in Fällen, in denen ein objektiv gerechtfertigtes Begehren mit hoher Wahrscheinlichkeit erfolgreich durchgesetzt werden könnte.

3 Wenn nun aber die Beschreitung des Rechtswegs unabhängig von der materiellen Berechtigung des streitgegenständlichen Rechtsanspruchs immer auch die Inkaufnahme eines mehr oder minder grossen (Kosten-)Risikos bedingt, so steht dies in einem gewissen Widerspruch zur konstitutionellen Rechtsweggarantie, die jedermann einen gangbaren Zugang $\mathrm{zu}$ wirksamem richterlichem Rechtsschutz verspricht. Tatsächlich ist das dem Zivilprozess immanente Prozesskostenrisiko eine Hürde, die der Rechtsuchende zu Beginn des Rechtswegs erst einmal zu überwinden hat. Die nachfolgenden Ausführungen beleuchten diese Hemmwirkung der Kosten der Rechtsverfolgung im Kontext des Anspruchs auf Zugang zum Recht.

\section{Anspruch auf Zugang zum Recht}

4 Die in Art. 29a BV1 verbriefte Rechtsweggarantie gewährt in prinzipieller Hinsicht einen grundrechtlichen Anspruch auf richterliche Beurteilung bei Rechtsstreitigkeiten. Demnach besteht zunächst ein Anrecht auf Zugang zu mindestens einer den Anforderungen von Art. $30 \mathrm{BV}$ genügenden Gerichtsinstanz ${ }^{2}$, die sich

1 Bundesverfassung der Schweizerischen Eidgenossenschaft vom 18. April 1999 (SR 101).

2 Die Verfassungskonformität eines Gerichts setzt voraus, dass dieses durch Gesetz geschaffen, zuständig, unabhängig und unparteiisch ist (Art. 30 Abs. 1 Satz 1 BV). 
mit der Streitsache befasst. 3 Die nämliche Behörde muss sodann zur umfassenden Überprüfung sämtlicher Rechts- und Sachverhaltsfragen befugt sein.4 Die Rechtsweggarantie eröffnet somit den Zugang zu einem Gericht mit voller Kognition in rechtlicher und tatsächlicher Hinsicht, führt jedoch darüber hinaus nicht zu einem Anspruch auf richterliche Nachprüfung durch ein höheres oder gar das Bundesgericht im Sinne einer Rechtsmittelgarantie. 5 Zumindest im Kontext zivilrechtlicher Ansprüche weitgehend analoge Garantien ergeben sich auch aus den supranationalen Menschenrechtspakten (Art. 6 Ziff. 1 EMRK 6 und Art. 14 Abs. 1 UNO-Pakt II7).

5 Weder dem verfassungs- noch dem menschenrechtlichen Anspruch auf Zugang zu einem Gericht bzw. - ganz allgemein ausgedrückt - zum Recht kommt derweil absolute Geltung zu. So hat das Bundesgericht wiederholt festgehalten, dass ein Rechtsweg insbesondere nur im Rahmen der jeweils geltenden Prozessordnung besteht und durch die üblichen Sachurteilsvoraussetzungen eingeschränkt wer-

Vgl. BGE 138 V 271 E. 3.1; Urteil des Bundesgerichts 1C_310/2009 vom 17. März 2010 E. 2.2.2; Botschaft über eine neue Bundesverfassung vom 20. November 1996 (BBl 1997 I 1) 502 f.

4 Nicht gewährleistet ist derweil die Kontrolle der Angemessenheit eines angefochtenen Akts; vgl. BGE 137 I 235 E. 2.5; Urteil des Bundesgerichts $1 \mathrm{C} \_310 / 2009$ vom 17. März 2010 E. 2.2.2; Botschaft BV (Fn. 3), 523.

5 Vgl. Bernhard Waldmann, Kommentar zu Art. 8, 29, 29a BV, in: Bernhard Waldmann/Eva Maria Belser/Astrid Epiney (Hrsg.), Bundesverfassung, Basler Kommentar, Basel 2015, Art. $29 a$ N 5.

6 Konvention vom 4. November 1950 zum Schutze der Menschenrechte und Grundfreiheiten (SR 0.101).

7 Internationaler Pakt über bürgerliche und politische Rechte vom 16. Dezember 1966 (SR 0.103.2). den kann. ${ }^{8}$ Solche Restriktionen dürfen aber nicht dazu führen, dass der Zugang zum Recht übermässig erschwert wird. Der grundsätzliche Anspruch auf richterlichen Rechtsschutz muss mit anderen Worten effektiv gewährleistet sein und darf nicht durch den Einbau verfahrensrechtlicher (faktischer oder formeller) Hemmschwellen, deren Überwindung dem Rechtsuchenden in der Praxis nicht oder kaum mehr zumutbar ist, seiner Wirkung beraubt werden. ${ }^{9}$ Daraus folgt auch, dass die Kosten der Gerichtsbarkeit keine über das Zumutbare hinausgehende prohibitive Wirkung entfalten dürfen. ${ }^{10}$

6 In die im Wesentlichen gleiche Richtung stösst auch die Strassburger Rechtsprechung zu Art. 6 Ziff. 1 EMRK, der in diesem Sinne ebenfalls keinen absoluten Zugang zum Gericht garantiert: Einschränkungen des Zugangsrechts sind demnach grundsätzlich zulässig, wenn sie (1.) einen legitimen Zweck verfolgen, (2.) die Verhältnismässigkeit zwischen eingesetztem Mittel und Zweck wahren und (3.) den Wesensgehalt - «the very essence» - des Rechts nicht beeinträch-

8 Vgl. Urteil des Bundesgerichts 1C_663/2012 vom 9. Oktober 2013 E. 6.2; BGE 137 II 409 E. 4.2; BGE 136 I 323 E. 4.3 m.w.H.; Urteil des Bundesgerichts 5P.319/2005 vom 9. November 2005 E. 4.1.

9 Vgl. Andreas Kley, Kommentar zu Art. 29a BV, in: Bernhard Ehrenzeller/Benjamin Schindler/Rainer J. Schweizer/Klaus A. Vallender (Hrsg.), Die schweizerische Bundesverfassung St.Galler Kommentar, 3. A., Zürich 2014, Art. $29 a$ N 8; Waldmann (Fn. 5), Art. $29 a$ N 13, 28.

10 Vgl. Urteil des Bundesgerichts 4P.315/2006 vom 22. Mai 2007 E. 2.3.4; Urteil des Bundesgerichts 4P.325/2006 vom 22. Mai 2007 E. 5. Derselbe Schluss ergibt sich im Übrigen auch aus dem Äquivalenzprinzip; vgl. dazu etwa BGE 106 Ia 249 E. 3a. 
tigen, mithin der effektive Zugang gewährleistet bleibt. ${ }^{11}$

7 Vor diesem Hintergrund erachtet der EGMR auch Gerichtskosten als zulässige Zugangsvoraussetzung, sofern sie nicht exzessiv sind und ohne Rücksicht auf die Vermögenslage der betreffenden Partei eingefordert werden.12 Der Staat muss demnach dafür Sorge tragen, dass dem Einzelnen der Zugang zum Gericht nicht aus wirtschaftlichen Gründen faktisch verwehrt wird, wobei er bei der Wahl der Mittel frei ist. ${ }^{13}$ Dabei kann das Zugangsrecht nicht nur durch vorprozessual erhobene, sondern auch durch nachträglich auferlegte Kosten verletzt werden.14

\section{Kostenrisiko und Zugang zum Recht}

\section{Vorbemerkung}

8 Das schweizerische Zivilprozessrecht begegnet der Problematik der potentiell rechtsschutzzugangsversperrenden Kraft

11 Vgl. Urteile des EGMR 72118/01 vom 15. November 2007 (Khamidov c. Russie), § 155; 38212/97 vom 30. Oktober 1998 (F.E. c. France), \& 44; 14003/88 vom 27. August 1991 (Philis c. Grèce), $\S 59 ; 8225 / 78$ vom 28. Mai 1985 (Ashingdane c. Royaume-Uni), § 57.

12 Vgl. Jochen Abraham Frowein/Wolfgang Peukert, Europäische MenschenRechtsKonvention, EMRK-Kommentar, 3. A., Kehl 2009, Art. 6 N 68 mit Verweis auf Urteil des EGMR 20656/03 vom 25. September 2007 (Loncke c. Belgique); Anne Peters/Tilmann Altwicker, Europäische Menschenrechtskonvention, 2. A., München 2012, $\S 19 \mathrm{~N} 39$.

13 Neben einer Kostenhilfe kommt etwa die Vereinfachung des Verfahrens in Frage, wodurch dem Rechtsuchenden das Prozessieren ohne kostspieligen Rechtsbeistand ermöglicht wird. Vgl. Urteil des EGMR 6289/73 vom 9. Oktober 1979 (Airey c. Irlande), § 26; ferner Urteil des EGMR 40031/98 vom 19. September 2000 (Gnahoré c. France), § 38.

14 Vgl. Urteil des EGMR 68490/01 vom 12. Juli 2007 (Stankov c. Bulgarie), § $53 \mathrm{f}$. der Prozesskosten primär mit dem Institut der unentgeltlichen Rechtspflege. Durch den Anspruch des Mittellosen auf Befreiung von den Gerichtskosten und auf Bestellung eines unentgeltlichen Rechtsbeistands gemäss Art. 117 ff. ZPO und Art. 29 Abs. 3 BV erscheint es als ausgeschlossen, dass ein Rechtsuchender bei ausgewiesenem Bedarf einzig aus wirtschaftlichen Gründen auf staatlichen Rechtsschutz verzichten muss. Prima vista ist damit dem grund- und konventionsrechtlichen Anspruch auf Zugang zum Recht Genüge getan - zumindest insoweit, als dieser Anspruch als Verbot tatsächlicher Rechtswegsperren, welche die Inanspruchnahme der Gerichtsbarkeit verunmöglichen, verstanden wird.

9 Indes wurde bereits dargelegt, dass die Rechtsweggarantie gemäss der Rechtsprechung des Bundesgerichts und des EGMR nicht erst dann verletzt ist, wenn der Zugang zum Gericht gänzlich versperrt bleibt, sondern bereits dann, wenn dieser in unzumutbarer Weise erschwert wird. Vor diesem Hintergrund ist mit Blick auf die Rechtsrealität festzustellen, dass in der Praxis aus Kostengründen vielfach auf die (gerichtliche) Durchsetzung von berechtigten Rechtsansprüchen verzichtet wird, obwohl die betreffenden Anspruchsinhaber durchaus über die notwendigen Mittel zur Prozessführung verfügen. Dies führt vor Augen, dass die drohenden Kosten der Rechtsverfolgung bzw. das mit der Prozessführung einhergehende Kostenrisiko eine spürbar abschreckende Wirkung auf die Rechtsuchenden zeitigen. Die Gründe hierfür liegen auf der Hand. 


\section{Zugangshemmender Effekt des Kostenrisikos}

Im Grundsatz ist davon auszugehen, dass die Entscheidung, einen Prozess anzustrengen, davon abhängt, ob der erwartete Erfolg den voraussichtlich damit einhergehenden Aufwand überwiegt, also eine gesamthafte Nutzenssteigerung erreicht wird.15 Der rational handelnde Rechtsuchende wird die Inanspruchnahme der staatlichen Gerichtsbarkeit daher in der Regel davon abhängig machen, ob die Wahrscheinlichkeit und das Ausmass des subjektiven Nutzens es rechtfertigen, das Risiko eines den ausbleibenden oder geminderten Erfolg überwiegenden Aufwand mit Blick auf die persönliche Situation im Einzelfall in Kauf zu nehmen. ${ }^{16}$ Auf der Aufwandseite sind hierbei die drohenden, mit der Prozessführung einhergehenden Kosten naheliegenderweise von überragender Bedeutung, weshalb der Faktor des Kostenrisikos von zentraler Wichtigkeit für die Entscheidung für oder wider die Anstrengung eines Prozesses ist.17 Je grös-

15 Vgl. dazu Richard A. Posner, Economic analysis of law, 9. A., New York 2014, 3 ff.

16 Diese Annahme bezieht sich auf den durchschnittlichen, rational denkenden Menschen und greift insofern zu kurz, als irrationale Verhaltensweisen im Rechtsalltag keineswegs eine Ausnahmeerscheinung darstellen.

17 Neben den Durchsetzungskosten sind namentlich die subjektive Bewertung des angestrebten Nutzens und der prozessualen Erfolgsaussichten sowie die individuelle Risikobereitschaft ausschlaggebend hinsichtlich des Entschlusses für bzw. des Verzichts auf die Prozessführung; vgl. dazu Martin Lorenz Kochheim, Die gewerbliche Prozessfinanzierung, Diss. Univ. Hamburg 2002, 41 ff.; Michael Adams, Kostenersatzrecht - ein Prozessordnungsvergleich aus ökonomischer Sicht, in: Peter Lewisch/Walter H. Rechberger (Hrsg.) 100 Jahre ZPO - Ökonomische Analyse des Zivilprozesses, Wien 1998, 225 ff., 234 f. Vgl. sodann die aussagekräftige (ökonomische) Analyse bei Moritz Dimde, Rechtsschutzzugang und Prozessfi- ser das Kostenrisiko hierbei ausfällt, umso eher wird der Rechtsuchende von der Einleitung eines Gerichtsverfahrens absehen.

11 Das Kostenrisiko als Inbegriff des nur in Grenzen prognostizierbaren finanziellen Prozessaufwands fungiert somit insofern als Hemmschwelle in Bezug auf den $\mathrm{Zu}-$ gang zum Recht, als es das Individuum von der gerichtlichen Geltendmachung eines rechtlichen Anspruchs abhalten kann. Dies gilt nicht nur hinsichtlich vermögensrechtlicher Streitigkeiten, wo die rein wirtschaftliche Betrachtungsweise tendenziell im Vordergrund steht, sondern kann auch in Belangen eine entscheidende Rolle spielen, in denen finanzielle Interessen nur bedingt oder gar nicht von Bedeutung sind ${ }^{18}$.

12 Das Kostenrisiko stellt in diesem Sinne kein immediates Rechtsweghindernis dar, wie dies etwa bei der Gerichtskostenvorschusspflicht (Art. 98 ZPO) der Fall ist. Gleichwohl vermag nach dem Gesagten die schiere Möglichkeit seiner Verwirklichung den Rechtsuchenden davon abzuhalten, den Rechtsweg zu beschreiten. Die dem Kostenrisiko innewohnende abschreckende Wirkung zeitigt damit eine mittelbare Beeinträchtigung des Zugangsrechts. ${ }^{19}$

nanzierung im Zivilprozess, Diss. HumboldtUniv. Berlin 2003, 94 ff.

$18 \mathrm{Zu}$ denken ist beispielsweise an rechtliche Auseinandersetzungen im Bereich des Persönlichkeitsschutzes.

19 Zur Beschränkung der Grundrechtsausübung durch Kostenübertragungen vgl. auch Stefan Leutert, Polizeikostentragung bei Grossveranstaltungen, Diss. Univ. Zürich 2005, 119 ff. Vgl. sodann Urteil des Verwaltungsgerichts Luzern vom 7. Mai 2013 (P 12 2), E. 6; Markus Schefer, Die Beeinträchtigung von Grundrechten - Zur Dogmatik von Art. 36 BV, Bern 2006, 28; Felix Uhl- 


\section{Auswirkungen in der Praxis}

13 Besonders ausgeprägt tritt die Hemmwirkung des Prozesskostenrisikos in denjenigen Fallkonstellationen in Erscheinung, in denen der betreffende Rechtsuchende zwar über die nötigen Mittel zur Prozessführung verfügt, das damit einhergehende Kostenrisiko aber so gross ist, dass ein negativer Prozessausgang (oder allenfalls die Verwirklichung des Inkassorisikos ${ }^{20}$ ) wenn nicht den finanziellen Ruin, so zumindest eine erhebliche Verschlechterung der wirtschaftlichen Situation zur Folge hätte. Die Inkaufnahme eines solchen Risikos erscheint auch bei günstigen Prozessaussichten regelmässig als untragbar. ${ }^{21}$

14 Diese Problematik ist unter der geltenden Kostenregelung im Zivilprozess evident. Die rechtsweghindernde Wirkung des Kostenrisikos wächst zwangsläufig mit der Höhe der drohenden Kosten und steht in Relation zum Nutzen, der mittels der gerichtlichen Rechtsdurchsetzung erlangt werden kann. ${ }^{22}$ Dabei zeigt die notgedrungen rein wirtschaftliche und damit messbare - Betrachtung der Rechtslage, dass das Kostenrisiko zuweilen in keinem Verhältnis zum durch das prozessuale Obsiegen angestrebten Erfolg steht. So gebietet die Vernunft dem Rechtsuchenden bei der Anrufung des Richters aufgrund der naturbedingten

mann, Das Willkürverbot (Art. 9 BV), Bern 2005, $\mathrm{N} 583$.

20 Unter Inkassorisiko ist vorliegend das Risiko der Uneinbringlichkeit des Prozessgewinns, der zugesprochenen Parteientschädigung und/oder des Ersatzanspruchs für vorgeschossene Gerichtskosten zu verstehen.

${ }^{21}$ Vgl. dazu auch Erich Fechner, Kostenrisiko und Rechtswegsperre - Steht der Rechtsweg offen?, in: JZ 11-12/1969, 349 ff., 350; Hans-Martin Pawlowski, Zur Funktion der Prozesskosten, in: JZ 7/1975, 197 ff., 197.

22 In diesem Sinne auch Adams (Fn. 17), 234 f.
Ungewissheit über den Verfahrensverlauf selbst bei vorzüglichen Prozessaussichten die Eventualität des Unterliegens nicht ausser Betracht zu lassen. Dabei wird er nicht selten zur Einsicht gelangen, dass der diesfalls drohende finanzielle Aufwand den möglichen Prozessgewinn durchaus übertreffen kann.

15 Dies lässt sich anhand eines konkreten Anwendungsbeispiels veranschaulichen: Ausgegangen wird von einem vermögensrechtlichen Rechtsstreit mit einem vergleichsweise tiefen aber für eine Person mit durchschnittlichem Einkommen gleichwohl beachtlichen - Streitwert von Fr. 40'0oo. Abgestellt wird beispielhaft auf die Situation im Kanton Zürich, womit die Gebührenverordnung des Obergerichts vom 8. September 2010 (GebV OG; LS 211.11) sowie die Verordnung über die Anwaltsgebühren vom 8. September 2010 (AnwGebV; LS 215.3) massgebend sind:

16 Zunächst ist die Gebühr für das Verfahren vor dem Friedensrichter zu berücksichtigen, die sich bei einem Streitwert von Fr. 40'0oo zwischen Fr. 420 und Fr. 615 bewegt ( 33 Abs. 1 GebV OG).

17 Für das Verfahren vor Bezirksgericht ist sodann von einer Grundgebühr über Fr. 4'750 auszugehen ( $\$ 4$ Abs. $1 \mathrm{GebV}$ $\mathrm{OG}$ ), die unter Umständen bis auf das Doppelte erhöht werden kann (§ 4 Abs. 2 GebV OG). Hinzu kommen gegebenenfalls weitere Gerichtskosten, insbesondere die Kosten der Beweisführung (vgl. dazu die Verordnung der obersten kantonalen Gerichte über die Entschädigung der Zeugen und Zeuginnen, Auskunftspersonen und Sachverständigen vom 11. Juni 2002 [LS 211.12]). Für die Entschädigung der 
anwaltlichen Vertretung der Gegenpartei ist mit einem Aufwand von Fr. 6'10o (inkl. MWST) zu rechnen (§ 4 Abs. 1 AnwGebV), die im Einzelfall jedoch auch erheblich höher ausfallen kann (vgl. § 4 Abs. 2 und $\S 11$ AnwGebV). Damit sind für das erstinstanzliche Verfahren ohne Berücksichtigung der mannigfaltig möglichen Mehrauslagen Prozesskosten von rund Fr. 11'ooo zu erwarten.

Einzubeziehen ist sodann die latente Eventualität eines Rechtsmittelverfahrens: Für ein allfälliges Berufungsverfahren vor Obergericht bemessen sich die Kosten grundsätzlich nach den für die Vorinstanz geltenden Massstäben, weshalb der Einfachheit halber von einer Verdoppelung der erstinstanzlichen Kosten auszugehen ist (vgl. $\S 12$ GebV OG und $\S 13$ AnwGebV). Bis zum Berufungsentscheid fallen somit (wiederum ungeachtet der Vielzahl an denkbaren kostensteigernden Umständen) Prozesskosten von ca. Fr. 22'00o an.

19 Folgt schliesslich noch ein Beschwerdeverfahren vor Bundesgericht, so ist dafür mit einer (interpolierten) Gerichtsgebühr von rund Fr. 2'700 zu rechnen (Art. 65 BGG i.V.m. Ziff. 1 des Tarifs für die Gerichtsgebühren im Verfahren vor dem Bundesgericht vom 31. März 2006 [SR 173.110.210.1]). Hinzu kommt die Parteientschädigung, wobei sich die Kosten des gegnerischen Rechtsanwalts auf etwa Fr. 5'ooo (inkl. MWST) belaufen dürften (Art. 68 BGG i.V.m. Art. 4 des Reglements über die Parteientschädigung und die Entschädigung für die amtliche Vertretung im Verfahren vor dem Bundesgericht vom 31. März 2006 [SR 173.110.210.3]).
20 Dieses Anwendungsbeispiel zeigt, dass die zivilprozessuale Durchsetzung einer Forderung über Fr. 40'ooo bei Inanspruchnahme der drei nationalen Instanzen entsprechend den vorstehenden Ausführungen Prozesskosten (bestehend aus den Gerichtskosten und der Parteientschädigung) von knapp Fr. 30'ooo zeitigt. Werden jetzt noch die eigenen Auslagen, namentlich die Vergütung des eigenen Rechtsbeistands, berücksichtigt, so übertrifft das Kostenrisiko den angestrebten Prozessgewinn bei weitem. Dabei gilt es zu bedenken, dass die vorgenommene Berechnung auf einer konservativen Schätzung beruht. Abhängig von den Umständen des Einzelfalls können die Kosten auch viel höher ausfallen (vorab gilt dies in komplexen Fällen, die einen grossen Zeitaufwand verursachen oder zusätzliche Verhandlungen bedingen sowie bei beträchtlichen Barauslagen der Gerichte für die Entschädigung von Sachverständigen u.Ä.). Diesfalls können die Rechtsverfolgungskosten sogar bereits im erstinstanzlichen Verfahren vor Bezirksgericht den Streitwert überschreiten. 23

21 Wenn aber das Kostenrisiko nicht deutlich niedriger ist als der in Aussicht stehende monetäre Nutzen, sondern diesem

23 Für Beispiele der Berechnung der Rechtsverfolgungskosten in Fällen mit höherem Streitwert vgl. Isaak Meier/Riccarda Schindler, Unerschwinglichkeit der Rechtsdurchsetzung - eine Verweigerung des Zugangs zum Gericht?, in: Walter Fellmann / Stephan Weber (Hrsg.), Haftpflichtprozess 2015, Zürich 2015, 29 ff., $34 \mathrm{ff}$. (Prozesskosten bei einem Streitwert von Fr. 1,5 Mio. in den Kantonen Zürich, Thurgau, Luzern, St.Gallen und Schwyz) sowie Markus Schmid, Unerschwinglichkeit der Rechtsdurchsetzung - die Fakten, in: Walter Fellmann/ Stephan Weber (Hrsg.), Haftpflichtprozess 2015, Zürich 2015, 13 ff., 16 ff. (Prozesskosten bei einem Streitwert bis Fr. 1 Mio. in den Kantonen Aargau, Basel-Stadt, Bern, Luzern und Zürich). 
in etwa die Waage hält oder ihn sogar überwiegt, so erzeugt es eine umso abschreckendere Wirkung gegenüber der potentiellen Prozesspartei. Dies gilt umso mehr, wenn die drohenden Kosten eine merkliche Verschlechterung der finanziellen Situation des Rechtsuchenden und gegebenenfalls der von ihm wirtschaftlich abhängigen Personen herbeizuführen vermögen.

\section{Folgerung: Kostenrisiko als fakti- sche Rechtswegsperre im Einzelfall}

Zunächst ist daran zu erinnern, dass die Rechtsweggarantie den nicht in unzumutbarer Weise erschwerten, effektiven Zugang zum Recht verspricht. Ob dieses Versprechen in Bezug auf die kostenbedingten Einschränkungen eingehalten wird, lässt sich - aufgrund der vielfältigen Einflussfaktoren - letztlich nur anhand des konkreten Einzelfalls und nicht in allgemeiner Weise aufgrund von Durchschnittswerten beurteilen. Doch kann auf dieser Grundlage zumindest das Potential des Kostenrisikos als nicht nur hemmende, sondern die Inanspruchnahme des richterlichen Rechtsschutzes unzumutbar erschwerende und damit faktisch vereitelnde Kraft erkannt werden.

$\mathrm{Zu}$ denken ist in diesem Zusammenhang an den Fall, in dem ein Rechtsuchender, der die Anforderungen der prozessualen Bedürftigkeit nur knapp nicht erfüllt, eine an sich berechtigte, aber schwierig zu beweisende Forderung von geringem Wert einklagen will. Unter derartigen Umständen erscheint die Prozessführung in Anbetracht der damit einhergehenden Risiken schlicht als unsinnig; aus Anwaltssicht wäre einem Mandanten $\mathrm{zu}$ seinem eigenen Schutz von der Rechts- verfolgung abzuraten. Dies ist nur ein stellvertretendes Beispiel für die mannigfaltigen Fallkonstellationen, in denen die Inkaufnahme des Prozesskostenrisikos und damit die Inanspruchnahme der staatlichen Rechtspflege für den Betroffenen wirtschaftlich nicht mehr tragbar sind.

24 Konkreter lässt sich mit Blick auf die dargestellten Risiken fragen, unter welchen Umständen ein «vernünftiger» Rechtsuchender bereit wäre, einen Prozess anzustrengen, dessen negativer Ausgang eine Gefährdung seiner wirtschaftlichen Existenz (und gegebenenfalls auch derjenigen, der von ihm abhängigen Personen) zur Folge hätte. Würde er als zweifacher Familienvater mit einem monatlichen Nettoeinkommen von Fr. 5'ooo einen Anspruch über Fr. 10'000 prozessual durchsetzen, wenn die (hypothetische) Erfolgswahrscheinlichkeit bei etwa 60 Prozent liegt und die drohenden Kosten bei einem Unterliegen im schlimmsten Fall den Streitwert sogar noch übersteigen? Ist die Inkaufnahme eines solchen Risikos zumutbar? Wie verhält es sich sodann bei modifizierten Umständen, wenn beispielsweise die Erfolgsaussichten günstiger sind, jedoch die Zahlungsfähigkeit der Gegenpartei bezweifelt werden muss? Solche Gedankenspiele führen unweigerlich zum Fazit, dass das Kostenrisiko die Anstrengung eines Prozesses auch bei Bestehen eines berechtigten Anspruchs oftmals als unwirtschaftliches Vorgehen erscheinen lässt.

25 Während sich finanziell besser gebettete Personen dieses Risiko dennoch erlauben können, so muss von weniger leistungsfähigen Parteien ab einem gewissen Punkt 
erwartet werden, dass sie - mitunter auch bei aussichtsreichen Ausgangssituationen - aus Rücksicht auf ihre finanzielle Lage von der Rechtsverfolgung absehen, da diese mit Blick auf die möglichen Negativfolgen ein unvernünftiges Risiko darstellen würde. Wenn das Anrufen des Richters aufgrund der drohenden Kosten aber als unvernünftiges, verantwortungsloses Verhalten abgetan wird, so ist solches dem Rechtsuchenden auch kaum mehr zumutbar. Diesfalls kommt dem Kostenrisiko nicht mehr bloss erschwerende, sondern verhindernde Wirkung zu: Der Zugang zum Recht wird zwar nicht tatsächlich verunmöglicht, aber indirekt durch die Aufbürdung eines schwerlich tragbaren Risikos erheblich eingeschränkt und dadurch weitgehend versperrt. 24

\section{Nutzen des zugangshemmenden Effekts des Kostenrisikos}

26 Die vorangehenden Ausführungen führen zum Schluss, dass die drohenden, den in Aussicht stehenden Nutzen möglicherweise übersteigenden Kosten die Rechtsverfolgung nicht nur in wenig aussichtsreichen Fällen als unwirtschaftlich erscheinen lassen können. Gerade bei (natürlichen wie juristischen) Personen mit vergleichsweise geringer finanzieller Leistungskraft kann dies zur Folge haben, dass auch die Durchsetzung an sich berechtigter Ansprüche die Inkaufnahme

$24 \overline{\text { Vgl. dazu auch Dimde (Fn. 17), } 152 \text { f.; Fechner }}$ (Fn. 21), 350 ff.; Erika Bokelmann, «Rechtswegsperre» durch Prozesskosten, in: ZRP 7/1973, 164 ff., 164 f.; Klaus Müller, Zur Problematik des Prozesskostenrisikos im Zivilprozess, in: JR 1/1987, 1 ff., 1; Kochheim (Fn. 17), 44; Pawlowski (Fn. 21), 197; Erwin Baier, Kostenrisiko und Grundgesetz, Diss. Univ. Erlangen-Nürnberg 1974, 26 f., 82; Arnd Herrmann, Probleme des Prozesskostenrisikos unter besonderer Berücksichtigung des Armenrechts, Diss. Univ. Kiel 1973, 20. unvernünftiger Risiken bedingt. Die Betroffenen werden diesfalls regelmässig und mit guten Gründen die bittere Pille des Rechtsverlusts schlucken und von der Anstrengung eines Prozesses absehen. Der Rechtsweg steht diesfalls wohl formell offen, doch erscheint seine Beschreitung als wirtschaftlich unzumutbar, womit er faktisch dennoch weitgehend abgeschnitten wird. Mit anderen Worten birgt das dem schweizerischen Zivilprozess innewohnende Kostenrisiko das Potential, unter gegebenen Umständen den Zugang zum Recht aufgrund seiner Abschreckungswirkung («chilling effect») übermässig und damit in unzulässiger Weise zu erschweren.

27 Im Kontext dieser prima vista wenig befriedigenden Feststellung darf indessen nicht negiert werden, dass die hemmende und bisweilen verhindernde Wirkung des Prozesskostenrisikos durchaus auch erhebliche Vorteile mit sich bringt. Die Entgeltlichkeit der Prozessführung verhindert etliche unnötige bis querulatorische Verfahren (und Verfahrenshandlungen);25 ebenso wird dadurch dem un-

25 Vgl. Benedikt A. Suter/Cristina von Holzen, Kommentar zu Art. 95 - 103 ZPO, in: Thomas SutterSomm/Franz Hasenböhler/Christoph Leuenberger (Hrsg.), Kommentar zur Schweizerischen Zivilprozessordnung (ZPO), 3. A., Zürich 2016, Art. $95 \mathrm{~N} \mathrm{1}$; Patrick Wamister, Die unentgeltliche Rechtspflege, die unentgeltliche Verteidigung und der unentgeltliche Dolmetscher unter dem Gesichtspunkt von Art. 4 BV und Art. 6 EMRK, Diss. Univ. Basel 1983, 3; Beat Ries, Die unentgeltliche Rechtspflege nach der aargauischen Zivilprozessordnung vom 18. Dezember 1984, Diss. Univ. Zürich 1990, 4; Viktor Rüegg/Michael Rüegg, Kommentar zu Art. 91 - 123 ZPO, in: Karl Spühler/Luca Tenchio/Dominik Infanger (Hrsg.), Schweizerische Zivilprozessordnung, Basler Kommentar, 3. A., Basel 2017, Art. 95 N 2; Botschaft zur Totalrevision der Bundesrechtspflege vom 28. Februar 2001 (BBl 2001 4202) 4239 f.; Pawlowski (Fn. 21), 198; Jürgen Riehl, Prozesskosten und die Inanspruchnahme der Rechtspflege, 
erwünschten Prozessieren «auf gut Glück» ein Riegel geschoben. Im Gegenzug würde der Wegfall der Kostenschranke wohl eine massive Mehr- bis Überbelastung der staatlichen Rechtspflege ${ }^{26}$ und damit einen wuchtigen Anstieg der Kosten der Justiz zur Folge haben, folglich eine erheblich zunehmende Strapazierung der öffentlichen Hand und damit im Endeffekt der Allgemeinheit der Steuerzahler bewirken.

Neben dem überflüssigen Ressourcenverschleiss spricht sodann die Tatsache gegen einen uneingeschränkten Zugang zum Gericht, dass es aus gesellschaftlicher Sicht sicherlich nicht erstrebenswert ist, dass jede Streitigkeit vor dem Richter endet. In diesem Zusammenhang bietet das Kostenrisiko einen Anreiz zur einvernehmlichen privaten Streitbeilegung und fördert die Vergleichsbereitschaft im Prozess. ${ }^{27}$

Diss. Univ. Giessen 2000, 69; Baier (Fn. 24), 173; zweifelnd offenbar Bokelmann (Fn. 24), 164, die das Fehlen eines - nach der hier vertretenen Ansicht überflüssigen - empirischen Nachweises dieser Annahme bemängelt. Vgl. dazu im Übrigen James W. Hughes / Edward Snyder, Litigation and Settlement under the English and American Rules: Theory and Evidence, in: J.L. \& Econ. 1/1995, 225 ff., 248, die aus ihrer Studie eine Korrelation zwischen der Höhe der Verfahrenskosten und der Qualität der Klagen herleiten.

26 In den Worten Benders droht diesfalls eine Arbeitslawine, welche die Gerichte mittels eines Rechtsstabs herkömmlichen Umfangs niemals bewältigen könnten (vgl. Rolf Bender, Einige Aspekte zu den Erfolgsbarrieren in der Justiz, in: RabelsZ 3-4/1976, 718 ff., 718).

27 Vgl. Rüegg/Rüegg (Fn. 25), Art. $95 \mathrm{~N} \mathrm{2}$; Suter/von Holzen (Fn. 25), Art. $95 \mathrm{~N}$ 1; Christel Stix, Gerichtliche und aussergerichtliche Durchsetzung ziviler Rechtsansprüche - Rechtlicher Vergleich und ökonomische Analyse, Diss. Univ. Frankfurt (Main) 1991, 223. Zum Einfluss der Kostenerstattungspflicht auf die Vergleichsbereitschaft vgl. Robert D. Cooter / Daniel L. Rubinfeld, Economic Analysis of Legal Disputes and Their Resolution, in: J. Econ. Lit. 3/1989, 1067 ff., 1075 ff.; Steven Shavell, Suit, Settlement,
29 Wohl noch gewichtiger sind die (positiven) Auswirkungen des Kostenrisikos mit Blick auf das Verhalten des potentiell Beklagten: Wer sich widerrechtlich verhält, muss mit einer Klage und der Last der mit dem Prozess einhergehenden Kosten rechnen; den Prozesskosten kommt insofern punitiver Charakter zu. ${ }^{28}$ Zöge das Unterliegen im Zivilverfahren indessen keine Kostenfolgen nach sich, so bliebe beispielsweise die Nichtbezahlung einer Schuld aus Sicht des Säumigen im besten Fall folgenlos, ${ }^{29}$ während im schlechtesten Fall lediglich die Vollstreckung der sowieso geschuldeten Forderung zuzüglich Verzugszins drohen würde. Dass diese Aussichten der Missachtung rechtlicher Verpflichtungen Vorschub leisten würde, liegt auf der Hand. Je bescheidener demnach das Prozesskostenrisiko ausfällt, desto geringer ist in diesem Kontext die Motivation zur freiwilligen Erfüllung von Rechtspflichten. ${ }^{0}$

3o Im Gegenzug schützt die hemmende Wirkung der Rechtsverfolgungskosten den potentiell Beklagten in gewissem Masse davor, ohne guten Grund zu gerichtlichen Auseinandersetzungen genötigt zu werden. Besonders zu erwähnen ist in diesem Zusammenhang die Verpflichtung der unterliegenden Partei, dem obsiegenden Prozessgegner in Form

and Trial: A Theoretical Analysis under Alternative Methods for the Allocation of Legal Costs, in: J. Legal Stud. 2/1982, 63 ff. sowie der Überblick bei Mark Schweizer, Prozesskostenerstattung und Wahrscheinlichkeit eines Vergleichs, in: Jusletter vom 13. April 2015, Rz 2 ff.

28 Vgl. dazu auch Baier (Fn. 24), $134 \mathrm{f}$.

29 Nämlich dann, wenn der Gläubiger auf die Durchsetzung seines Anspruchs verzichtet.

3o Vgl. dazu auch Pawlowski (Fn. 21), 198; Marc André Jacot, Die Kosten der Rechtsverfolgung als Schranke für den Rechtsuchenden, Diss. Univ. Zürich 1978, 100. 
der Parteientschädigung Ersatz für die diesem angefallenen Parteikosten zu leisten. ${ }^{11}$ Zwar gilt es zu bedenken, dass die zugesprochene Parteientschädigung nicht sämtliche durch die Prozessführung anfallenden Parteikosten und - insbesondere bei Streitigkeiten mit einem tiefen Streitwert - regelmässig nicht einmal den vertraglich vereinbarten Honoraranspruch der anwaltlichen Vertretung zu decken vermag. ${ }^{22}$ Gleichwohl erfüllt die drohende Kostenerstattungspflicht als Teilaspekt des Prozesskostenrisikos eine wesentliche Funktion zum Schutz des Einzelnen davor, zu Unrecht in Anspruch genommen zu werden: Entfiele die Pflicht zur Leistung der Parteientschädigung im Unterliegensfalle, so sänke infolgedessen einerseits das Kostenrisiko und damit die Hemmschwelle für die Einleitung ungerechtfertigter Verfahren. Andererseits würde dadurch die Missbrauchsgefahr wachsen; die blosse Androhung prozessualer Schritte dürfte vermehrt als Druckmittel zur Erlangung unberechtigter Vorteile (Vergleichszahlungen etc.) Verwendung finden, wenn auch die obsiegende Partei in jedem Fall ihre eigenen Parteikosten tragen müsste. 33

31 Art. 95 Abs. 1 und 3 i.V.m. 106 Abs. 1 ZPO.

32 Vgl. dazu etwa Meier/Schindler (Fn. 23), 49; Martin Sterchi, Kommentar zu Art. 91-116 ZPO, in: Berner Kommentar, Schweizerische Zivilprozessordnung, Band I, Art. 1-149 ZPO, Bern 2012, Art. 95 N 12 ff.; Benjamin Schumacher, Prozessfinanzierung - Erfolgshonorierte Fremdfinanzierung von Zivilverfahren, Diss. Univ. Zürich 2015, N 35; Pascal Grolimund, Lücken und Tücken bei Rechtsschutzversicherungen, in: Walter Fellmann/Stephan Weber (Hrsg.), Haftpflichtprozess 2015, Zürich 2015, 119 ff., 121.

33 Veranschaulichen lässt sich die Problematik an der Situation in den USA. Die Geltung der sog. American rule, wonach die Prozessparteien unabhängig vom Prozessausgang ihre eigenen Anwaltskosten tragen müssen, scheint eine erhöhte Gefahr des Missbrauchs in Form der Androhung
31 Die metaphorische Prozesskostenkeule dient den vorangehenden Ausführungen entsprechend gewichtigen öffentlichen Interessen, als sie in gewissem Umfang dem unnötigen Ausufern der Zahl vor Gericht ausgetragener Auseinandersetzungen entgegenwirkt, wodurch die Belastung und die Kosten der Justiz eingedämmt werden. Gleichzeitig leistet sie damit einen Beitrag an die Wahrung des Rechtsfriedens, da sie einen mittelbaren Anreiz zunächst zur Verhinderung und in der Folge zur einvernehmlichen Bereinigung von Streitigkeiten bietet.

\section{Würdigung}

32 Das Kostenrisiko der Rechtsverfolgung zwingt den Rechtsuchenden dazu, sich die Beschreitung des Rechtswegs wohl zu überlegen. Dieser Abwägung von Kosten und Nutzen der Prozessführung fällt zuweilen die Geltendmachung berechtigter Anliegen zum Opfer, was für sich gesehen mit Blick auf den grund- und menschenrechtlich garantierten Anspruch auf Rechtsschutz zumindest bedenklich erscheint. Dies gilt vorab für diejenigen Fälle, in denen die objektiv gerechtfertigte Inanspruchnahme der staatlichen Rechtspflege infolge der drohenden Kosten die wirtschaftliche Existenz des Rechtsuchenden gefährden würde und infolgedessen als in unzumutbarem Masse risikobehaftetes Vorgehen erscheint.

bzw. Anhebung grundloser Klagen als Druckmittel («legal blackmail») zur Folge zu haben; vgl. dazu etwa Corinne Widmer Lüchinger, Schweizerisches und US-amerikanisches Haftpflichtrecht: Konvergenz/Divergenz, in: ZBJV 2015, 545 ff., 560; Lorenz Lauer, Kollektiver Rechtsschutz im Schweizer Privatrecht, in: BJM 2017, 173 ff., 180 f.; David A. Root, Attorney Fee-Shifting in America: Comparing, Contrasting, and Combining the "American Rule» and «English Rule», in: Ind. Int'l \& Comp. L. Rev. 3/2005, 583 ff., 584 und 605 f. m.w.H. 
Problematisch ist jedoch bereits die keineswegs seltene Konstellation, in welcher die mögliche Verwirklichung des (auch bei durchaus berechtigter Prozessführung lauernden) Kostenrisikos einzelfallbezogen zwar keine erhebliche Einbusse in der finanziellen Situierung bedingen würde, die Anstrengung eines Verfahrens aber insgesamt als unwirtschaftlich und damit wenig sinnvoll erscheinen lässt. Das Kostenrisiko zeitigt insofern stets eine (mittelbare) Beeinträchtigung des Zugangsrechts.

So unbefriedigend diese Situation zunächst anmutet, handelt es sich dabei dennoch um ein wohl in Kauf zu nehmendes, notwendiges Übel. Es ist anzunehmen, dass die Zahl der durch die abschreckende Wirkung des Kostenrisikos vermiedenen unnötigen und damit «gesellschaftlich unerwünschten» Prozesse überwiegt. Insgesamt dürften die aufgezeigten Vorteile der geltenden Kostenregelung deren Nachteile übertreffen. In diesem Lichte hat die dargelegte Einschränkung des Zugangsrechts hinsichtlich des öffentlichen Interesses insbesondere an einer funktionierenden Rechtspflege durchaus ihre Berechtigung.

34 Mit Blick auf die aufgezeigte Notwendigkeit einer Kostenschwelle ist wohl auch die Zurückhaltung des Bundesgerichts 34 und des EGMR bei der Annahme einer Verletzung des Zugangsrechts im Zusammenhang mit der Höhe der Prozesskosten zu erklären.

34 Vgl. dazu etwa die Urteile des Bundesgerichts 4P.315/2006 vom 22. Mai 2007 sowie 4P.325/2006 vom 22. Mai 2007.
35 In der bundesgerichtlichen Rechtsprechung findet sich soweit ersichtlich keine vertiefte Auseinandersetzung mit der Frage der zugangsbeschränkenden Wirkung der Entgeltlichkeit der Rechtspflege. Aus der Praxis zur unentgeltlichen Rechtspflege ergibt sich immerhin, dass eine unzumutbare Beschränkung der Rechtsweggarantie aufgrund von Rechtsverfolgungskosten dann anzunehmen ist, wenn der Rechtsuchende nicht über die notwendigen Mittel für die effektive Inanspruchnahme der staatlichen Rechtspflege verfügt.35 Dementsprechend besteht diesfalls mitunter gestützt auf Art. 29a BV ein justiziabler Anspruch auf Kostenhilfe. Darüber hinaus hat das Bundesgericht zwar eingeräumt, dass unverhältnismässig hohe Prozesskosten auch im Falle nicht mittelloser Rechtsuchender den Zugang zum Gericht zu verletzen vermögen, doch setzt es die Hürde für eine solche Annahme (zumindest implizit) sehr hoch an. ${ }^{6}$ Daraus folgt, dass Kosten, die der Rechtsuchende zwar bewältigen könnte, die aber die Prozessführung auch bei aussichtsreicher Ausgangslage als unvernünftiges Risiko erscheinen lassen, in den Augen der höchsten Richter grundsätzlich nicht bzw. nur in Extremfällen als unzulässige Zugangsbeschränkung erscheinen. Dementsprechend existiert auch kein Fall, in dem das Bundesgericht in einer konkreten Prozesskostenauferlegung unabhängig von der Frage nach einem Anspruch auf unentgeltliche Rechtspflege eine Verletzung

35 So bereits BGE 57 I 337 E. 3; vgl. sodann statt vieler Urteil des Bundesgerichts 4A_325/2015 vom 9. Februar 2016 E. 4.1; BGE 140 III 12 E. 3.3.1; BGE 139 III 396 E. 4.1; BGE 135 I 1 E. 7.1; BGE 120 Ia 14 E. 3d.

36 Vgl. Urteil des Bundesgerichts 4P.315/2006 vom 22. Mai 2007 E. 2.3.4; Urteil des Bundesgerichts 4P.325/2006 vom 22. Mai 2007 E. 5. 
des Rechts auf Zugang zum Gericht erkannt hätte.

Keinen weitergehenden Schutz gegen die zugangsbeschränkende Wirkung des Prozesskostenrisikos gestützt auf den Anspruch auf Zugang zum Gericht entnimmt bis anhin der EGMR Art. 6 Ziff. 1 EMRK. Der Gerichtshof hat sich mit dieser Frage - mit einer ersichtlichen Ausnahme, die betreffend einen Zivilprozess zwischen zwei Privatparteien indes von geringer Aussagekraft ist 37 - (noch) nicht explizit auseinandergesetzt. Gleichwohl ist festzustellen, dass er bereits die Anforderungen an die Notwendigkeit einer Prozesskostenhilfe für Bedürftige höher ansetzt, als dies das Bundesgericht tut. 38 Vor diesem Hintergrund verbietet sich die Annahme, dass gestützt auf die Strassburger Rechtsprechung mit Blick auf hohe Prozesskosten ein umfangreicherer Zugangsschutz besteht.

37 Entsprechend auch den vorstehenden Ausführungen zeigt die Analyse der (spärlichen) Rechtsprechung zum Verhältnis der Prozesskostenhöhe zur Rechtsweggarantie, dass nach Auffassung sowohl des Bundesgerichts als auch des EGMR der Zugang zum Gericht zwar nicht in unzumutbarer Weise erschwert sein darf, die Anforderungen an eine Unzumutbarkeit in diesem Zusammenhang indessen offensichtlich sehr hoch angesetzt sind. Gestützt auf diese Praxis bieten Art. 29a BV und Art. 6 Ziff. 1 EMRK lediglich in stossenden Einzelfällen ein justiziables Mittel gegen übermässig hohe Prozesskosten.39 Im Umkehrschluss

37 Vgl. Urteil des EGMR 68490/01 vom 12. Juli 2007 (Stankov c. Bulgarie).

38 Vgl. Urteil des Bundesgerichts 5A_85/2007 vom 17. April 2007 E. 3.1; BGE 119 Ia 264 E. 3.

39 In diesem Sinne auch Meier/Schindler (Fn. 23), 54 . bedeutet dies, dass die zugangsbeschränkende Wirkung des mit den hierzulande anfallenden Rechtsverfolgungskosten im Zivilprozess einhergehenden Kostenrisikos grundsätzlich als zulässig erachtet wird, obwohl dadurch - nach hier vertretener Auffassung - nicht nur in Ausnahmefällen der Zugang zum Recht erheblich erschwert wird.

38 Wenngleich die scheinbar weitgehende höchstrichterliche Akzeptanz der zugangsbeschränkenden Wirkung der Rechtsverfolgungskosten zumindest in einem gewissen Widerspruch zum Postulat des nicht in unzumutbarer Weise erschwerten, effektiven Zugangs zur Gerichtsbarkeit steht, so hat sie dennoch grundsätzlich ihre Berechtigung. Die gegenteilige Auffassung hätte gezwungenermassen die Notwendigkeit einer merklichen Senkung der Gerichtsgebühren bzw. erheblich weitergehender Kostenhilfen zur Folge. Solches lässt sich im Sinne einer optimalen Erhältlichkeit von Rechtsschutz leicht fordern, wenn die damit verbundenen negativen Auswirkungen auf die Rechtspflege ausser Acht gelassen werden. Die Zugangserleichterung durch die Herabsetzung der Kostenhürde im Zivilprozess würde gleichermassen einen entsprechenden Wegfall des Nutzens des hemmenden Effekts des Kostenrisikos provozieren. Weitere (erhebliche) Kostenerleichterungen im Zivilprozess erscheinen vor diesem Hintergrund zwar unter dem Gesichtspunkt der Rechtsweggarantie geboten, doch sprechen gleichzeitig gewichtige öffentliche Interessen - Funktionieren der Rechtspflege, Förderung des Rechtsfriedens, Entlastung der Staatsfinanzen - gegen einen solchen Schritt. Im Lichte dieser Interessenkollision erscheint es als nach- 
vollziehbar, wenn die dargestellte (mittelbare) Beeinträchtigung des Anspruchs auf Zugang zur Rechtspflege zugunsten der Wahrung der erwähnten öffentlichen Interessen im Sinne der Herstellung praktischer Konkordanz ${ }^{40}$ zumindest stillschweigend als zulässig erachtet wird.

39 In Anbetracht der bundesgerichtlichen Vorgaben und unter Berücksichtigung der Strassburger Rechtsprechung lassen sich Art. 29a BV (und Art. 6 Ziff. 1 EMRK) nach dem Gesagten im Hinblick auf grundlegende kostenbezogene Erleichterungen des Zugangs zum Recht über das Recht auf unentgeltliche Rechtspflege hinaus keine konkreten subjektivrechtlichen Ansprüche entnehmen.41 Mit der Ausnahme stossender Einzelfälle sind das geltende Kostenrecht und die damit verbundenen Zugangsdefizite für eine Vielzahl von Rechtsuchenden folglich mit der Rechtsweggarantie nach höchstrichterlichem Verständnis vereinbar.

Aus diesem Schluss folgt nicht, dass der Status quo bedenkenlos beibehalten werden kann. Selbst wenn dieser im Lichte der geltenden Auslegung des Anspruchs auf Zugang zum Recht nicht grundsätzlich unhaltbar ist, wurde doch aufgezeigt, dass - wenn nicht flächendeckend, dann doch in bestimmten Konstellationen - eine Verbesserung der Zugangsmöglichkeiten durch die Relativierung der Kostenschranke zumindest wünschenswert wäre. Mit Blick auf den programmatischen Gehalt von Art. $29 a$ i.V.m. Art. $35 \mathrm{BV}$ ist der Gesetzgeber gehalten, Änderungsmög-

\footnotetext{
40 Vgl. dazu BGE 139 I 16 E. 4.2.2 sowie die a.a.O. angeführten Hinweise auf Lehre und Rechtsprechung.

41 Ähnlich Meier/Schindler (Fn. 23), 54.
}

lichkeiten im Kostenrecht hin zu einer zugangsfreundlicheren Regelung zu prüfen und gegebenenfalls umzusetzen. Ziel einer solchen Revision wäre entsprechend den vorangehenden Ausführungen eine Mässigung der kostenbedingten Zugangshemmnisse insbesondere für objektiv gerechtfertigte Anliegen, die - namentlich im Hinblick auf die angeführten, auf dem Spiel stehenden öffentlichen Interessen nicht zugleich noch schwerwiegendere Nachteile nach sich zieht, wie das - als Extrembeispiel - etwa bei einer allgemeinen Kostenlosigkeit der Inanspruchnahme der Justiz der Fall wäre. 